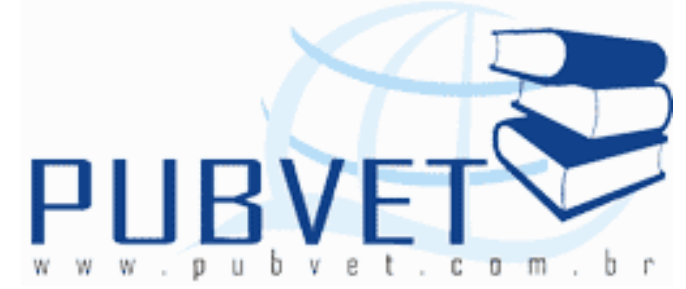

PUBVET, Publicações em Medicina Veterinária e Zootecnia.

\title{
Eritrograma de fêmeas suplementadas com minerais durante três períodos reprodutivos
}

\author{
Vanessa Veronese Ortunho ${ }^{1}$, Wilmar Sachetin Marçal ${ }^{2}$, Mara Regina Stipp \\ Balarin $^{2}$, Nelson Parizotto Junior ${ }^{3}$
}

${ }^{1}$ Aluna de pós graduação Stricto sensu da Universidade Estadual de Londrina, Paraná, Brasil, e-mail: vanessaverort@yahoo.com.br

2 Docente da Universidade Estadual de Londrina, Departamento de Clínica Veterinária-CCA

${ }^{3}$ Aluno de pós-graduação Stricto sensu da Universidade Estadual de Londrina.

\section{Resumo}

Com os objetivos de detectar se a utilização de diferentes formulações minerais poderia alterar os valores do eritrograma, introduzir na literatura valores de sanguíneos de fêmeas criadas no Paraná e comparar as alterações sanguíneas que ocorrem durante o período seco, gestação e 10 dias do pósparto, realizou-se este trabalho. Foram utilizadas 30 fêmeas ovinas da raça Suffolk, as quais foram separadas aleatoriamente em dois grupos de 15 fêmeas: um grupo recebeu sal mineral comercial contendo componentes inorgânicos e o outro grupo recebeu sal mineral comercial contendo alguns minerais orgânicos na forma de carboaminofosfoquelato. O sistema adotado foi o confinamento e o sal mineral foi fornecido ad libitum. As colheitas de sangue foram mensais e iniciaram um mês antes do início da estação de monta, que 
iniciou quando as fêmeas estavam com aproximadamente 8 meses de idade. $\mathrm{A}$ suplementação mineral interferiu somente no HCM e a presença da gestação não interferiu em nenhum dos parâmetros analisados.

Palavras-chave: Eritrograma. Suplementação mineral. Ovelhas.

\title{
Eritrogram of sheep that was received different minerals in 3 periods
}

\begin{abstract}
The objectives of this study were to compare the effects of supplementation with organic and inorganic minerals in eritrograma, to compare the changes that occurs in 3 reproductives periods and provide to literature values of this parameter in the females created in Paraná State, was made this experiment. The objects of the study were 30 Suffolk ewes randomly distributed in two groups of 15 females. A control group received inorganic commercial mineral salt and the challenge group received commercial mineral salt containing some organic minerals in the carboaminofosfoquelate form. The adopted system was the confinement and the salt was supplied ad libitum. All animals had blood collected monthly and the first collect was one month before the breeding season started, when the females were approximately 8 months old. The mineral supplementation only influencied one parameter and the presence of pregnancy didn't influence the parameters.
\end{abstract}

Keywords: Eritrogram. Mineral supplementation. Sheep.

\section{Introdução}

A importância da hematologia como meio semiológico, auxiliando os veterinários a estabelecerem diagnósticos, firmarem prognósticos e acompanharem os tratamentos das inúmeras enfermidades que atingem os animais domésticos é reconhecida e consagrada mundialmente. Entretanto, para que esses objetivos possam ser alcançados e utilizados na plenitude é fundamental o conhecimento dos valores de referência do hemograma dos 
ORTUNHO, V.V. et al. Eritrograma de fêmeas suplementadas com minerais durante três períodos reprodutivos. PUBVET, Londrina, V. 7, N. 3, Ed. 226, Art. 1499, 2013.

animais sadios, bem como dos fatores causadores de suas variações, como nível de estresse, sexo dos animais, fase reprodutiva, raça dos animais, entre outros (VIANA et al., 2002).

Há uma concordância quase unânime entre os pesquisadores que os animais criados sob diferentes condições ambientais, climáticas e de manejo podem apresentar evidentes variações dos elementos constituintes do hemograma. Assim, os valores obtidos para os animais criados em uma região não podem ser considerados, sem uma adequada avaliação, como padrão de referência fora dessa região (VIANA et al., 2002). Por isso, Eshratkhah, et al., (2011) e Braun; Trumel; Bézille, (2010), recomendam que os valores de referência sejam produzidos e validados por cada laboratório, pois cada um utiliza uma metodologia diferente e geralmente analisa sangue de animais da região próxima de onde situa o laboratório, facilitando assim, a interpretação dos resultados.

Sabe-se que a nutrição é um fator que altera os parâmetros sanguíneos e que é um dos itens que mais onera a produção, por isso nos últimos anos ela tem despertado interesse nos pesquisadores, na indústria e nos produtores com o uso dos minerais orgânicos nas misturas ofertadas para os animais (PEIXOTO et al., 2005). Eles são formados por um mineral ligado a uma molécula orgânica e quando processados recebem o nome de quelatos, formando estruturas com características próprias, como alta biodisponibilidade, tamanho pequeno e ausência de cargas elétricas (SPEARS, 1996); (KELLOGG, KEGLEY, 2002) e (PAL et al., 2010).

Para avaliarem os efeitos do cobre orgânico na hematologia de ovinos, Eckert et al., (1999) pesquisaram 30 ovelhas da raça Rambouillet, sendo que 15 fêmeas receberam $\mathrm{CuSO}_{4}$ e as demais; proteinato de cobre. Durante o experimento, foram oferecidos 3 níveis deste mineral: 10, 20 e $30 \mathrm{mg} / \mathrm{kg}$. 0 sangue foi colhido nos dias 28 e 73 após o início do experimento e os autores não encontraram diferença estatística entre os grupos no eritrograma.

Mohri et al., (2011) pesquisaram 16 cordeiros Baloochi com 70 dias de idade que foram divididos em 2 grupos, sendo que um deles recebeu vitamina 
E e selenito de sódio injetados na dose de $0,2 \mathrm{mg} / \mathrm{kg}$ e o outro grupo recebeu a mesma dose de solução salina, os autores não encontraram diferença na concentração de hemoglobina e na quantidade de linfócitos.

Sabendo que a gestação também é um fator de variação Brito et al., (2006) realizaram um trabalho para estudar o perfil hematológico de 14 ovinos leiteiros da raça Lacaune criados em confinamento na Serra Gaúcha. Amostras de sangue foram colhidas de ovelhas vazias; gestantes e lactantes. Os parâmetros hematológicos não mostraram diferença nos períodos fisiológicos estudados, com exceção dos neutrófilos segmentados, que aumentaram com o avanço da gestação.

Obidike, Aka, Okafor (2009) acompanharam 12 ovelhas West African Dwarf durante o terço final da gestação e início da lactação e não foram encontradas diferenças no hematócrito, contagem de eritrócitos, no volume corpuscular médio e na concentração de hemoglobina, quando compararam os valores sanguíneos pré-parto e pós-parto.

Sabendo que diferentes raças podem ter os parâmetros sanguíneos alterados, Tibbo et al., (2005), analisaram a influência da raça nos parâmetros hematológicos de 377 ovinos saudáveis criados na Etiópia pertencentes a 3 raças nativas. A concentração de hemoglobina, hemoglobina corpuscular média, concentração da hemoglobina corpuscular média das raças nativas foram maiores que os das raças exóticas, enquanto que houve uma diminuição no número de linfócitos e aumento do número de neutrófilos, conforme a idade dos animais aumentava.

Sabendo-se que animais submetidos ao estresse têm o seu desempenho comprometido, Mc Manaus et al., (2008) pesquisaram a influência da temperatura nas ovelhas criadas no Brasil. Foram utilizadas 30 fêmeas secas Santa Inês, 10 Santa Inês x Bergamascia e 10 Bergamascia. Foram feitas 6 colheitas em dias diferentes e nestes dias eram realizadas 2 colheitas, uma de manhã e outra a tarde, sendo que entre o intervalo, os animais ficavam sob o sol e com água à vontade. Os valores de $\mathrm{Hct}, \mathrm{He}, \mathrm{Hgb}$, foram menores na Bergamascia, enquanto que WBC, VCM e CHCM não apresentaram diferenças 
entre as raças. Os autores concluíram que os animais da raça Santa Inês foram os que menos sofreram com o estresse e mostraram-se mais adaptados ao clima do país.

Devido à escassez de trabalhos que avaliem o hemograma de ovinos criados no Brasil e a urgência destes valores serem conhecidos realizou-se esta pesquisa que teve como objetivos: determinar valores do hemograma de animais sadios criados no norte do Paraná, Brasil, que estejam no período seco, gestantes e lactantes e também, verificar se a suplementação mineral altera os parâmetros hematológicos dos ovinos.

\section{Material e Métodos}

O experimento foi realizado numa propriedade rural localizada no município de Prado Ferreira, $23^{\circ} 02^{\prime} 22^{\prime \prime}$ de latitude Sul, $51^{\circ} 26^{\prime} 32^{\prime \prime}$ de longitude Oeste e $651 \mathrm{~m}$ de altitude, norte do Paraná, Brasil (IPARDES, 2007).

Foram utilizadas 30 fêmeas ovinas da raça Suffolk, as quais foram separadas aleatoriamente em dois grupos de 15 fêmeas: um grupo recebeu sal mineral comercial inorgânico e o outro grupo recebeu sal mineral comercial contendo alguns minerais orgânicos, na forma de carboaminofosfoquelato. Para que não houvesse mistura entre os lotes, foi colocado colar nos animais, sendo que cada grupo, usou uma cor. Optou-se por trabalhar com sal mineral comercial para que pudesse ser simulado o que realmente ocorre no campo.

As fêmeas iniciaram o consumo dos sais aos 4 meses de idade, ao desmame, com pesos iniciais de $20,26 \pm 4,54 \mathrm{~kg}$ para as fêmeas que receberam sal mineral orgânico e 21,9 \pm 4,9 kg para as fêmeas que receberam sal mineral inorgânico. O sistema adotado foi o confinamento e a alimentação consistiu de ração comercial (23,04\% Proteína Bruta), cujo consumo era de 2 kg/animal por dia; cana picada, que era oferecida todos os dias; água e sal mineral; fornecidos à vontade nos cochos.

As composições dos sais minerais para ovinos utilizados encontram-se detalhadas na Tabela 1, a seguir. 
Tabela 1 - Fórmula do sal mineral orgânico e inorgânico que foi fornecido ao grupo experimental e controle, em níveis de garantia por $\mathrm{kg}$ do produto.

\begin{tabular}{|c|c|c|}
\hline Elemento & $\begin{array}{c}\text { Formulação Mineral } \\
\text { Orgânica }\end{array}$ & $\begin{array}{c}\text { Formulação Mineral } \\
\text { Inorgânica }\end{array}$ \\
\hline Cálcio & $120 \mathrm{~g}$ & $140 \mathrm{~g}$ \\
\hline Fósforo & $87 \mathrm{~g}$ & $60 \mathrm{~g}$ \\
\hline Sódio & $147 \mathrm{~g}$ & $136 \mathrm{~g}$ \\
\hline Enxofre & $* 18 \mathrm{~g}$ & $5 g$ \\
\hline Cobre & $* 590 \mathrm{mg}$ & $150 \mathrm{mg}$ \\
\hline Cobalto & $* 40 \mathrm{mg}$ & $90 \mathrm{mg}$ \\
\hline Cromo & *20mg & - \\
\hline Ferro & $* 1.800 \mathrm{mg}$ & - \\
\hline Iodo & $80 \mathrm{mg}$ & $180 \mathrm{mg}$ \\
\hline Manganês & $* 1.300 \mathrm{mg}$ & $400 \mathrm{mg}$ \\
\hline Selênio & $* 15 \mathrm{mg}$ & $13 \mathrm{mg}$ \\
\hline Zinco & $* 3.800 \mathrm{mg}$ & $3.000 \mathrm{mg}$ \\
\hline Molibdênio & $300 \mathrm{mg}$ & - \\
\hline Flúor (máx.) & $870 \mathrm{mg}$ & $600 \mathrm{mg}$ \\
\hline Magnésio & - & $6 g$ \\
\hline Cloro & - & $216 \mathrm{~g}$ \\
\hline Lisina & - & $200 \mathrm{mg}$ \\
\hline Metionina (máx.) & - & $40 \mathrm{mg}$ \\
\hline Tirosina & - & $82 \mathrm{mg}$ \\
\hline Solubilidade do & $95 \%$ & $95 \%$ \\
\hline \multicolumn{3}{|l|}{ Fósforo em Acido } \\
\hline Cítrico a $2 \%$ (mín) & & \\
\hline
\end{tabular}

Em março de 2008 quando os animais estavam com aproximadamente 8 meses; 35,33 \pm 4,46 kg grupo orgânico e 37,2 $\pm 6,45$ kg grupo inorgânico, foram colocados em estação de monta, a qual teve duração de 3 meses, e para que não houvesse interferência do macho, houve rodízio mensal na cobertura. Após 2 meses de seu término, foi realizado o exame de ultrasonografia para verificação da quantidade de ovelhas prenhes no rebanho, as quais não foram separadas do lote inicial para que não fosse introduzida outra variável. 
A primeira colheita de sangue foi realizada 1 mês antes das ovelhas entrarem em estação de monta, fevereiro de 2008. A segunda colheita foi realizada no dia que a estação de monta foi iniciada e as outras colheitas foram realizadas conforme o estado reprodutivo da fêmea, tabela 2 e 3.

Após o parto e com os dados da ultrasonografia, calculou-se em que mês as ovelhas ficaram prenhes, então, para elas adotou-se o calendário de colheita que está explicado na tabela 2.

Tabela 2 - Calendário de colheita de sangue das fêmeas que emprenharam durante o experimento.

\begin{tabular}{cc}
\hline Colheitas & Realizada \\
\hline 1 & 1 mês antes do início da estação de monta (fev. 2008) \\
2 & dia do início da estação de monta (março 2008) \\
3 & 10 mês de gestação \\
4 & 20 mês de gestação \\
5 & 30 mês de gestação \\
6 & 40 mês de gestação \\
7 & 50 mês de gestação \\
8 & 10 dias do pós-parto \\
\hline
\end{tabular}

Para as fêmeas que não emprenharam, o calendário de colheita de sangue está descrito na tabela 3 .

As colheitas de sangue foram realizadas após jejum over-night e através de venopunção da jugular utilizando o sistema a vácuo com agulhas BD Vacutainer ${ }^{\circledR}$ descartáveis $22 \mathrm{G} \mathrm{X} \mathrm{1",} \mathrm{adaptador} \mathrm{para} \mathrm{tubos} \mathrm{de} \mathrm{colheita} \mathrm{e} \mathrm{tubos}$ de $10 \mathrm{ml}$ sem anticoagulante. O transporte dos tubos até o laboratório foi realizado em estantes verticais, sendo que o conjunto estante- tubos foi colocado numa inclinação de aproximadamente $45^{\circ}$ para facilitar a separação do soro.

As amostras foram analisadas no mesmo dia da colheita e o eritrograma e a contagem do leucócitos foram feitas no aparelho $\mathrm{MS}_{4}$. As amostras foram 
analisadas no Laboratório de Patologia Clínica do Hospital Veterinário da Universidade Estadual de Londrina.

Tabela 3 - Calendário de colheita de sangue das fêmeas que não emprenharam durante o experimento.

\begin{tabular}{cc}
\hline Colheitas & Realizada \\
\hline 1 & 1 mês antes do início da estação de monta (fev. 2008) \\
2 & dia do início da estação de monta (março 2008) \\
3 & abril 2008 \\
4 & maio 2008 \\
5 & junho 2008 \\
6 & julho 2008 \\
7 & agosto 2008 \\
8 & setembro 2008 \\
\hline
\end{tabular}

Os dados obtidos foram avaliados através do programa SAEG (UFV, 2007). Foram realizadas análises de variância, testes de Tukey, regressão linear e múltipla dos parâmetros sanguíneos, tendo-se calculado as interações entre tipo de sal ofertado com presença de prenhez (TXP), tipo de sal ofertado com dias de colheita (TXD) e presença de prenhez com dias de colheita (PXD). Optou-se por fazer estudo da regressão até $3^{\circ} \mathrm{grau}$, para facilitar o estudo e a comparação entre os dados encontrados na literatura. Também se calculou no mesmo programa, pelo teste Qui-Quadrado, os dados de taxa de prenhez.

\section{Resultados e Discussão}

Durante a estação de monta, obteve-se 6 fêmeas prenhes no grupo que recebeu suplementação mineral inorgânica e 2 no grupo que recebeu suplementação com minerais orgânicos, segundo o teste Qui-Quadrado, não houve diferença estatística na taxa de prenhez. Acredita-se que esta taxa pode ter sido influenciada pelo consumo da cana, que é pobre em vitamina $A$, pelo baixo peso das fêmeas no início da estação de monta e por serem nulíparas. 
Para facilitar a análise dos dados, os grupos foram denominados de T para tipo de sal consumindo e $\mathrm{P}$ para presença ou não de prenhes, no experimento ficando as seguintes combinações:

-TOPO fêmeas que receberam sal inorgânico e que não ficaram prenhes,

-TOP1 fêmeas que receberam sal inorgânico e que emprenharam,

-T1P0 fêmeas que receberam sal orgânico e que não ficaram prenhes

-T1P1 fêmeas que receberam sal orgânico e que emprenharam.

Os resultados obtidos estão apresentados na Tabela 4.

Observa-se, através da tabela 4, que o tipo de sal ofertado durante o experimento, somente interferiu nos valores médios de HCM, tendo as fêmeas que receberam sal orgânico maior valor deste parâmetro; 43,04 mg/dl apresentado maiores valores deste parâmetro que as fêmeas do outro grupo; $40,33 \mathrm{mg} / \mathrm{dl}$.

Este trabalho concorda com, Eckert et al. (1999) que não encontraram diferença estatística no $\mathrm{He}$, Hct e $\mathrm{Hb}$ quando avaliaram o cobre na forma orgânica e inorgânica.

Nenhum dos parâmetros analisados foram afetados pela presença ou não de gestação, concordando com Brito et al. (2006) que ao pesquisarem a variação do perfil hematológico em ovinos leiteiros, no período seco, gestação e lactação da raça Lacaune criados em confinamento na Serra Gaúcha, não encontraram alterações significativas entre os períodos estudados nos parâmetros hematológicos.

Este trabalho concorda também com Obidike, Aka, Okafor (2009) que ao acompanharem as alterações hematológicas de ovelhas West African Dwarf durante o terço final da gestação e lactação não encontraram diferença no Hct, $\mathrm{He}, \mathrm{VCM}, \mathrm{Hgb}$.

Ovelhas da raça Suffolk criadas nas mesmas condições que as do experimento são capazes de no período seco, gestação e 10 dias de lactação de manterem os parâmetros hematológicos compatíveis com a produção e saúde. 
Pode ser observado, na tabela 4, que as interações calculadas, tipo de sal com dias de colheita, presença de prenhez com dias de colheita e a interação do tipo de sal ofertado com a prenhez, também não apresentaram diferença estatística.

Tabela 4- Médias observadas do eritrograma e do número de leucócitos, em função do tipo de sal, prenhes e dias de colheita

\begin{tabular}{|c|c|c|c|c|c|c|c|}
\hline Fontes de variação & $\mathrm{He}$ & $\mathrm{Hgb}$ & Hct & VCM & HCM & $\mathrm{CHCM}$ & WBC \\
\hline \multicolumn{8}{|l|}{ Tipo de sal ( $\mathrm{T}$ ) } \\
\hline Sal inorgânico (0) & 10,69 & 8,28 & 32,31 & 39,69 & $13,06 b$ & 32,96 & 8,56 \\
\hline Sal orgânico (1) & 10,87 & 7,97 & 32,23 & 40,87 & $13,62 \mathrm{a}$ & 32,96 & 8,62 \\
\hline \multicolumn{8}{|l|}{ Prenhes (P) } \\
\hline Ausente (0) & 10,94 & 8,10 & 32,29 & 40,30 & 13,51 & 33,13 & 8,54 \\
\hline Presente (1) & 10,64 & 8,14 & 32,26 & 40,27 & 13,18 & 32,80 & 8,65 \\
\hline \multicolumn{8}{|l|}{ Dias de Colheita } \\
\hline 1 & 10,19 & 8,91 & 29,68 & 33,55 & 11,43 & 34,50 & 6,91 \\
\hline 2 & 10,67 & 9,01 & 30,70 & 34,23 & 11,55 & 34,25 & 5,51 \\
\hline 3 & 11,52 & 9,99 & 34,06 & 34,16 & 10,52 & 31,20 & 10,38 \\
\hline 4 & 9,44 & 8,77 & 30,08 & 34,41 & 10,60 & 31,16 & 9,40 \\
\hline 5 & 10,63 & 6,83 & 31,52 & 46,17 & 15,54 & 33,78 & 9,09 \\
\hline 6 & 11,94 & 7,81 & 36,06 & 46,20 & 16,48 & 33,23 & 8,75 \\
\hline 7 & 11,00 & 7,21 & 33,26 & 46,22 & 15,26 & 33,05 & 10,96 \\
\hline 8 & 10,31 & 6,74 & 31,67 & 46,33 & 15,31 & 33,20 & 11,76 \\
\hline Médias Gerais & 10,78 & 8,12 & 32,27 & 40,28 & 13,34 & 32,96 & 8,60 \\
\hline \multicolumn{8}{|l|}{ Significância F } \\
\hline Tipo & NS & NS & NS & NS & NS & NS & NS \\
\hline Prenhes & NS & NS & NS & NS & NS & NS & NS \\
\hline Interação TXP & NS & NS & NS & NS & NS & NS & NS \\
\hline Interação TXD & NS & NS & NS & NS & NS & NS & NS \\
\hline Interação PxD & NS & NS & NS & NS & NS & NS & NS \\
\hline $\begin{array}{l}\text { Coeficiente de } \\
\text { variação }\end{array}$ & 23,84 & 15,64 & 15,91 & 3,72 & 13,06 & 5,28 & 35,60 \\
\hline
\end{tabular}


ORTUNHO, V.V. et al. Eritrograma de fêmeas suplementadas com minerais durante três períodos reprodutivos. PUBVET, Londrina, V. 7, N. 3, Ed. 226, Art. 1499, 2013.

\section{Conclusões}

Este estudo simulou o que ocorre nos parâmetros sanguíneos das ovelhas em vários períodos produtivos e reprodutivos das propriedades rurais do Brasil, que utilizam as formulações minerais utilizadas no experimento.

Esta pesquisa mostrou a necessidade da realização de estudos com minerais orgânicos que avaliem o hemograma dos animais suplementados, mostrou a necessidade da realização de pesquisas que avaliem os animais criados no país para que a realização do monitoramento dos animais seja facilitada, podendo assim ser realizada a adoção de medidas profiláticas para evitar perdas reprodutivas e produtivas, aumentando assim a produtividade.

Este estudo é importante por enriquecer o meio científico mostrando os valores do perfil eritrocitário de animais criados no Norte do Paraná e que poderão ser utilizados em outros estudos, como comparação.

Pode-se concluir com este experimento que as formulações minerais ofertadas para as fêmeas estudadas somente influenciaram os valores de HCM, enquanto que a presença da gestação não alterou nenhum dos parâmetros analisados. As interações calculadas não apresentaram diferença estatística para os parâmetros analisados.

\section{Referências}

BRAUN, J.P.; TRUMEL, C.; BÉZILLE, P. Clinical biochemistry in sheep: A selected review. Small Ruminant Research, France, v.92, p10-18, 2010.

BRITO, A.M., et al. Composição do sangue e do leite em ovinos leiteiros do sul do Brasil: variações na gestação e na lactação. Ciência Rural, Santa Maria, v.36, n.3, p.942-948, maijun, 2006.

ESHRATKHAH, B. et al. Relationship between the level of plasma insulin and lipidprofile in Iranian fat-tailed sheep. Comparative Clinical Pathology, Iran, v.20, n.3, p.223-226, 2011.

ECKERT, G. E. et al. Copper status of ewes fed increasing amounts of copper from copper sulfate or copper proteinate. Journal of Animal Science, Texas, v. 77, p. 244-249, 1999.

INSTITUTO PARANAENSE DE DESENVOLVIMENTO ECONOMICO E SOCIAL - IPARDES. Área, altitude e coordenadas geográficas, segundo os municípios do Paraná. In: Anuário estatístico do Estado do Paraná. Curitiba, 2007. Disponível em:

<http://www.ipardes.gov.br/anuario_2007/1territorio/tab1_1_1.htm> Acesso em: 20 out. 2008. 
KELLOGG; D.W., KEGLEY, E.B. Organic-Chelated Minerals. Feed supplements, p. 981-996, V[ ], 2002.

MC MANAUS et al.Heat tolerance in Brazilian sheep: Physiological and blood parameters. Trop Anim Health Prod, Brasília. 2008

MOHRI, M. et al. Parenteral Selenium and Vitamin E Supplementation to Lambs: Hematology, Serum Biochemistry, Performance, and Relationship with Other Trace Elements. Biological Trace Element Research, Iran. v.139, p.308-316, 2011.

OBIDIKE, I.R.; AKA, L.O.; OKAFOR, C.I. Time-dependant peri-partum haematological, biochemical and rectal temperature changes in West African dwarf ewes. Small Ruminant Research, Nigeria. v. 82, p.53-57, 2009.

PAL, D. T. et al. Effect of copper- and zinc-methionine supplementation on bioavailability, mineral status and tissue concentrations of copper and zinc in ewes. Journal of Trace Elements in Medicine and Biology, India, v. 24, p. 89-94, 2010.

PEIXOTO, P.V., et al. Princípios de suplementação mineral em ruminantes. Pesquisa Veterinária Brasileira, Brasil. v.25, ed.3, p.195-200, 2005.

SAEG . Sistema para Análises Estatísticas, Versão 9.1: Fundação Arthur Bernardes - UFV Viçosa, 2007.

SPEARS, J. W. Organic trace minerals in ruminant nutrition. Animal Feed Science and Technology, Amsterdam, v. 58, n. 1/2, p. 151-163, 1996.

TIBBO, M. et al. Factors affecting haematological profiles in three indigenous Ethiopian sheep breeds. Compedendiun Clinical Pathologic, London, v. 13, p. 119-127, 2005.

VIANA, R.B. et al. Influência da gestação e do puerpério sobre o leucograma de caprinos da raça Saanen, criados no Estado de São Paulo. Brazilian Journal Veterinarian Research animal Science, São Paulo. v. 39, n.4, p. 196-201, 2002. 